\title{
Smart Lamp or Security Camera? Automatic Identification of loT Devices
}

\author{
Thomsen, Mathias Dahl; Giaretta, Alberto; Dragoni, Nicola
}

Published in:

Proceedings of the $12<$ sup $>$ th $</$ sup $>$ International Networking Conference

Link to article, DOI:

10.1007/978-3-030-64758-2_7

Publication date:

2020

Document Version

Peer reviewed version

Link back to DTU Orbit

Citation (APA):

Thomsen, M. D., Giaretta, A., \& Dragoni, N. (2020). Smart Lamp or Security Camera? Automatic Identification of IoT Devices. In Proceedings of the 12 International Networking Conference (pp. 85-99). Springer. Lecture Notes in Networks and Systems Vol. 180 https://doi.org/10.1007/978-3-030-64758-2_7

\section{General rights}

Copyright and moral rights for the publications made accessible in the public portal are retained by the authors and/or other copyright owners and it is a condition of accessing publications that users recognise and abide by the legal requirements associated with these rights.

- Users may download and print one copy of any publication from the public portal for the purpose of private study or research.

- You may not further distribute the material or use it for any profit-making activity or commercial gain

- You may freely distribute the URL identifying the publication in the public portal 
See discussions, stats, and author profiles for this publication at: https://www.researchgate.net/publication/344490696

\section{Smart Lamp or Security Camera? Automatic Identification of IoT Devices}

Conference Paper · October 2020

CITATIONS

0

3 authors, including:

(f) Alberto Giaretta

29 PUBLICATIONS 253 CITATIONS

SEE PROFILE

Some of the authors of this publication are also working on these related projects:

Project Dynamic Reconfiguration of Business Workflows View project

Project E-care@home View project
READS

62

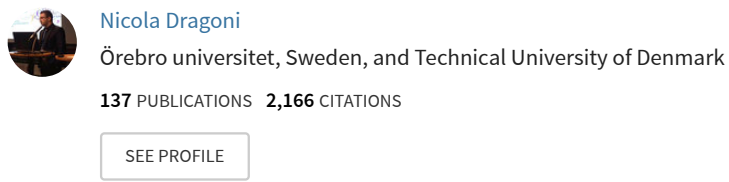




\title{
Smart Lamp or Security Camera? Automatic Identification of IoT Devices
}

\author{
Mathias Dahl Thomsen ${ }^{1}$, Alberto Giaretta ${ }^{20000-0001-9293-7711]}$, and \\ Nicola Dragoni ${ }^{1,2}[0000-0001-9575-2990]$ \\ 1 DTU Compute, Technical University of Denmark, Kongens Lyngby, Denmark \\ mdahlthomsen@hotmail.com, ndra@dtu.dk \\ 2 Centre for Applied Autonomous Sensor Systems (AASS), Örebro University, \\ Örebro, Sweden alberto.giaretta@oru.se
}

\begin{abstract}
The tsunami of connectivity brought by the Internet of Things is rapidly revolutionising several sectors, ranging from industry and manufacturing, to home automation, healthcare and many more. When it comes to enforce security within an IoT network such as a smart home, there is a need to automatically recognise the type of each joining devices, in order to apply the right security policy. In this paper, we propose a method for identifying IoT devices' types based on natural language processing (NLP), text classification, and web search engines. We implement a proof of concept and we test it against 33 different IoT devices. With a success rate of $88.9 \%$ for BACnet and $87.5 \%$ for MUD devices, our experiments show that we can efficiently and effectively identify different IoT devices.
\end{abstract}

Keywords: Internet of Things · device identification · profiling · natural language processing · text classification.

\section{Introduction}

With the increased popularity of the Internet of Things (IoT) and a rapidly expanding market, there is a growing demand for new IoT devices. On the one hand, this creates an opportunity for companies and start-ups to cover such demand, capitalise on this trend, and improve cooperation. In 2017 alone, 2888 North American startups managed to attract investments for $\$ 125$ billion. All combined, these 2888 startups grew up to $\$ 613$ billion and 95 of them managed to grow up to $\$ 1$ billion each. Last, investors play a fundamental role in transferring knowledge to and among startups [14].

On the other hand, the massive market demand pressures manufacturers to provide better features than competitors, quicker, and for lower prices. Unfortunately, this led many manufacturers to overlook the security of their devices, while focusing on more apparent features. As a result, many IoT devices currently operating are insecure, easy to penetrate, and to exploit for malicious activities [7. For example, botnet malwares recruit devices for striking Distributed Denial of Service (DDoS) attacks. Using this technique, in 2016 the 
Mirai malware took advantage of unprotected IoT devices and performed the largest DDoS attack ever recorded [5].

IoT devices specialise on a small set of tasks, collaborating with other devices for providing complex services (e.g., a set of sensors in a smart home). One critical point is that current devices do not specify which entities they collaborate with, in order to function properly 9 . This results in substrata of hidden behaviours and communications, hard to manage and reconcile with networks' security requirements. Expressing behaviours is a crucial aspect of identity management. As pointed out by Roman et al. [19], identity management is not only a problem of binding unique IDs with devices, but also of describing the core features of such devices.

Protocols and standards, such as Building Automation and Control networks (BACnet) [11] and Manufacturer Usage Descriptions (MUD) [13, partially address this issue. For instance, MUD devices explicitly describe their requirements through a MUD file. A network must be equipped with a node (the MUD Controller) which verifies MUD files and stores the network Access Control List (ACL). MUD is limited to domains, ports, and protocols used by IoT devices for their communications. This makes hard for network admins to reason about categories of devices, and to setup concise and intelligible network policies.

Following the line of explicit behavioural description, Giaretta et al. 9010 have recently proposed to apply the Security-by-Contract paradigm $(\mathrm{S} \times \mathrm{C})$ to IoT. In a nutshell, IoT devices store a contract which describes their securityrelevant behaviour, easy to check and verify against network security policies stored on a Fog node. In the past, $\mathrm{S} \times \mathrm{C}$ paradigm has been successfully applied to different fields, ranging from pervasive mobile computing [6], through web applications, to multi-application smart cards.

All these efforts share a common drawback: what happens if a device does not carry the behavioural description required by the network it is joining? For example, an IoT device might be MUD-compliant, but not $\mathrm{S} \times \mathrm{C}$-compliant. In the name of compatibility, it is desirable to allow devices to join the network with a minimal set of permissions, according to the nature of the devices themselves. This raises the problem of identifying devices' type. How can a network node, such as a Fog node controlling a smart home, discern an indoor lamp from a smart lock?

Contribution. In this paper, we propose an approach for identifying IoT devices, and we apply it to MUD and BACnet devices. In particular, we describe a technique that identifies devices' types by means of text classification algorithms and natural language processing (NLP). First, we perform experiments in order to identify which combination of algorithms identify devices best. Then, we evaluate our proof of concept (PoC), focusing on accuracy and speed. We also analyse the downsides of our implementation, and how to amend them.

Outline of the Paper. In Section 2 we motivate our work. In Section 3 we detail the necessary steps to identify an IoT device. In Section 4 we discuss a first implementation of our PoC, and in Section 5 we evaluate its performance. In 
Section 6 we highlight the potential legal issues of our system and how they can be amended. Also, we show that the second PoC shows better detection rates than the first version. In Section 7 we discuss benefits and shortcomings of our work and in Section 8 we give a brief overview of related work. Last, we wrap up our contribution in Section 9

\section{Motivation}

Let us assume that we add an IoT indoor lamp to our local network. It is reasonable to suppose that a lamp interacts with motion sensors, so that the light can be turned on and off, depending on our presence. It is equally reasonable to suppose that an indoor lamp does not interact with external security cameras.

The entire reasoning is based on the nature of the device, a smart lamp. It is irrelevant that the lamp is manufactured by Philips or Samsung, or that it is Model A7 or Model T1. There are cases where specific policies are necessary for specific devices, while other cases require a reasoning based on the task the devices perform (e.g., lighting up a room). Most protocols and paradigms, such as BACnet and MUD, do not enforce any information regarding the nature of the device.

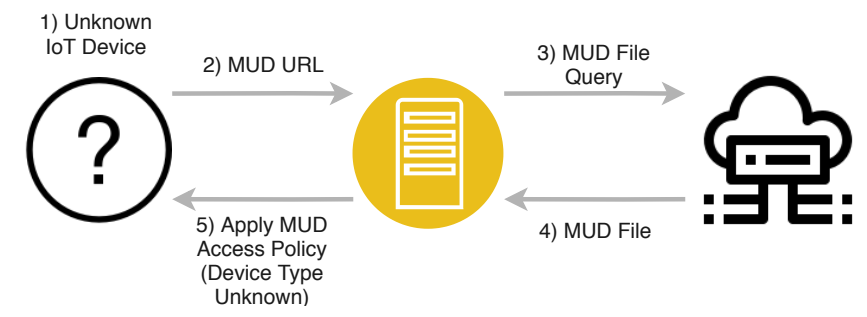

Fig. 1. Basic MUD architecture.

This is clearer by looking at the basic MUD architecture shown in Fig. 1. In short, the MUD device provides a MUD URL to the MUD Controller. In turn, the controller queries the URL, downloads the MUD file, and verifies it. If everything is correct, it creates a new access policy for the IoT device and then allows it to the network. The device evaluation is based on the fact that the manufacturer defined and signed a MUD file. No step of the MUD paradigm requires to know which device it deals with. This makes hard for network administrators to define security policies at the category level. How can we enforce a lamp-policy on our smart lamp, if our system cannot tell a lamp from a camera?

In Fig. 2 we show an example of how device identification could work with MUD and help administrators to apply policies according to categories. Once that MUD file has been retrieved, the Fog Node extracts and organises the relevant information. The Fog Node then feeds the data to a web search engine, 
gathers more information about the device, and combines everything together. The identification phase takes place and the network is now able to enforce security policies predefined for the appropriate category. If no policies have been defined for the category, or the identification attempt fails, the Fog Node can still apply the MUD file as-is.

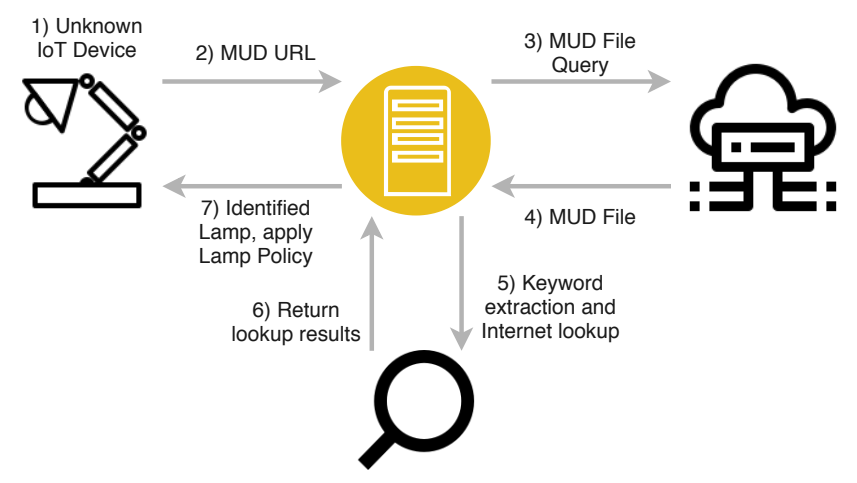

Fig. 2. MUD architecture integrated with our proposal.

We can apply our approach to different protocols and paradigms. For example, BACnet is a communications protocol for automated control systems, such as heating, ventilating, and air-conditioning control (HVAC). Each BACnet compliant device carries an object which describes its capabilities and relevant data. Similarly to what we have previously shown for MUD, in Fig. 3 we extract relevant information from a BACnet object, feed it to a web search engine, and process the results in order to identify the type of device.

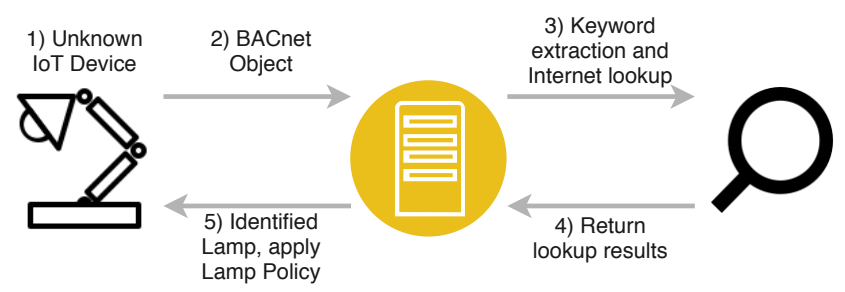

Fig. 3. BACnet integrated with our proposal.

\section{Device Classification}

Network traffic analysis is not suitable for quickly identifying IoT devices. Capturing and analysing pcap traces can require many hours of unrestricted in- 
teractions. This is not desirable, if such classification is necessary for enforcing network security policies, when a device joins the network.

In this paper, we propose a device classification approach which is composed of two main parts: the information retrieval and the text classification. As shown in Fig. 4, the first part (phase 1 and phase 2, in red) is responsible for extracting relevant texts from the device information fields. This part heavily depends on the IoT protocol stack, therefore it cannot be generalised for every IoT device. Phase 3, coloured in green in Fig. 4 is responsible for extracting relevant keywords from the texts (phase 3A), feeding them to web search engines and extracting the search results (phase 3B), and finally classifying the device (phase $3 \mathrm{C})$.
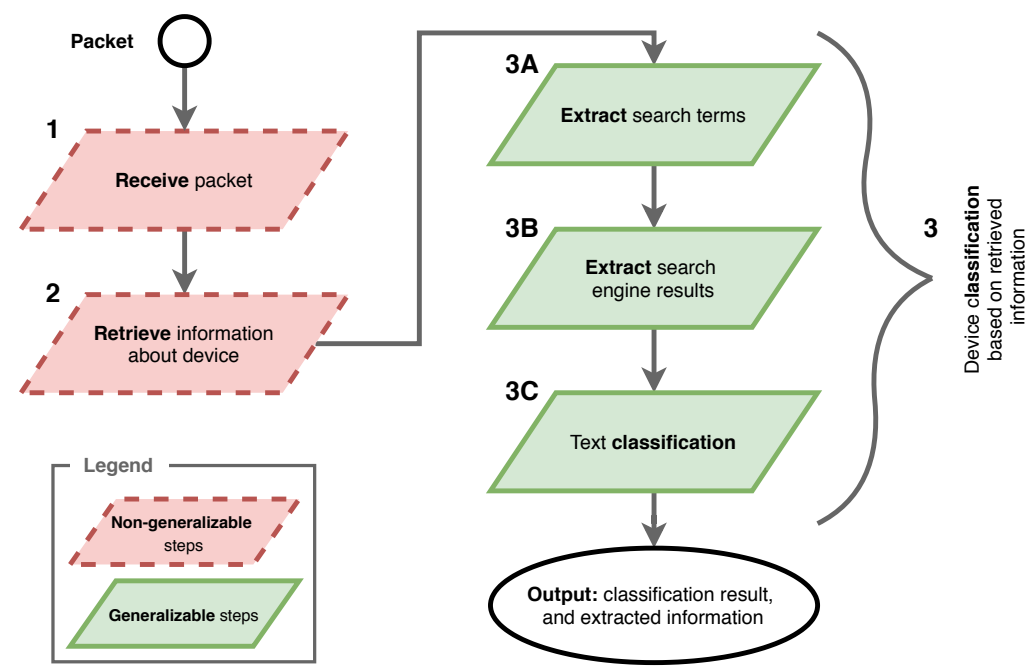

Fig. 4. Overview of a device profiling steps. The red steps cannot be generalised, since they depend on the specific protocol stack. The green steps, all part of the text classification phase, are generalisable.

In our vision, our implementation would run on a Fog node within a local network. Due to their computing power and their capability of offloading tasks to the Cloud layer [18] 15], Fog nodes are central elements for different frameworks, such as $\mathrm{S} \times \mathrm{C}[9$. In particular, our solution would rely on the upper Cloud layer (we refer the reader to the architectural model in [15]) to gather relevant information and extract the device type.

Even though it is out of the scope of this work, we highlight that this technique could fuel other interesting applications within other frameworks (such as $\mathrm{S} \times \mathrm{C}$ ). For example, the gathered data could provide some information about devices behaviour and enable the framework to create tailored contracts. Else, the framework could store predefined contracts for each IoT device type (e.g., 
lamp, camera, smart plug, etc.), and assign the correct one to the profiled device, based on the classification result. In any case, it is fundamental to correctly identify the device type.

\section{Proof of Concept}

In this Section, we describe the PoC we developed in Python for assessing our approach. The source code and the data sets are publicly available on GitHub 3 .

In Fig. 4 we noted that the initial steps cannot be generalised. With regard to the information retrieval step, we have to narrow down our scope and choose relevant standards and protocols. For general consumer IoT devices we focus on the Manufacturer Usage Description (MUD) standard. For industrial IoT devices, we take into consideration the BACnet protocol. The overall approach for extracting information is therefore different.

MUD files contain various fields that provide relevant information for detecting the device types. Most notably, the systeminfo field contains a 60 characters description of the device. The field is free text, so the content varies depending on the file creator. However, we can parse this field and extract important keywords to identify the device type. This is the only field used for extracting keywords for MUD capable devices. BACnet devices are represented by virtual entities called objects, which describe various properties. The most general object, the device object, contains text fields about vendor, model, and functionalities exhibited by the device. Similarly to MUD, we can extract keywords from these texts. In particular, vendor and model fields were the ones we used for extracting BACnet devices keywords.

There are differences in the keywords that can be extracted from the MUD files and BACnet objects. It is not defined what specific information is specified in systeminfo, whereas for the BACnet device object it is known that the fields will contain specific information about the vendor and what the model of the device is. For the purpose of this implementation, both the fields extracted from the MUD files and the BACnet objects are simply used as is without any attempt to discard irrelevant text from the field.

To gather as much information as possible, we feed the extracted keywords to Google and Bing. Our goal is to increase the amount of information at our disposal, so that we improve our chances of identifying the devices. Obtained all the relevant text, we can start the classification phase.

\subsection{Classification of Device Type}

In our work, we have considered various text classification models and algorithms for identifying the device type. However, classification models cannot understand free text. We need to convert the text to feature vectors. This step is called feature extraction. As we show in Table 1 we evaluated different NLP

\footnotetext{
${ }^{3}$ Publicly released at: https://github.com/MDThomsen/DBAC-Device-Detection
} 
approaches, such as Term Frequency (TF) and Term Frequency-Inverse Document Frequency (TF-IDF). We also evaluated various configurations of N-grams and custom Lemmatizers.

Table 1. Tested NLP approaches for feature extraction

\begin{tabular}{|c|c|c|c|c|}
\hline Test & Precision & Avg Recall Avg $F_{1}$ & Score & Avg Support \\
\hline $\mathrm{TF}$ & 0.88 & 0.75 & 0.76 & 11.7 \\
\hline $\begin{array}{l}\text { TF } \\
+ \text { Bigram }\end{array}$ & 0.86 & 0.75 & 0.75 & 11.1 \\
\hline $\begin{array}{l}\text { TF } \\
+ \text { Lemmatizer }\end{array}$ & 0.80 & 0.71 & 0.70 & 10.2 \\
\hline TF-IDF & 0.91 & 0.83 & 0.85 & 11.5 \\
\hline $\begin{array}{l}\text { TF-IDF } \\
+ \text { Bigram }\end{array}$ & 0.89 & 0.82 & 0.83 & 11.3 \\
\hline $\begin{array}{l}\text { TF-IDF } \\
+ \text { Lemmatizer }\end{array}$ & 0.90 & 0.82 & 0.83 & 11.3 \\
\hline doc2vec & 0.78 & 0.40 & 0.47 & 20.0 \\
\hline
\end{tabular}

Our tests were based on a Support Vector Machine (SVM) classification model, used as a baseline for each test. The dataset used for training and testing the feature extraction methods was based on approximately 100 different texts, manually created. Each text was assigned to 1 out of 10 different categories of general consumer IoT devices. We performed a $\mathrm{k}$-fold test (where $\mathrm{k}=8$ ), and for each fold we calculated precision, recall, and $\mathrm{F}_{1}$ score. Therefore, $F_{1}$ Score Avg is not calculated over Precision Avg and Recall Avg, but is the average of all $\mathrm{F}_{1}$ scores, previously calculated. TF-IDF scored better than TF, with respect to precision, recall, and $\mathrm{F}_{1}$ score. Using bigrams to extend the contextual information did not improve the accuracy of neither TF nor TF-IDF. Performing extra preprocessing of the text with a lemmatizer did not provide any gains in accuracy; this shows that additional modelling or preprocessing are unnecessary, for this specific instance. For implementing our PoC, we opted for TF-IDF.

Similar to what we have done for feature extraction approaches, we compared different text classification algorithms. In particular, we considered Logistic Regression, Support Vector Machines, Neural Networks, and Random Forests. For Logistic Regression, we considered 2 models. One based on the LibLinear implementation 8, the other one based on stochastic gradient descent (sgd) [17. For Support Vector Machines, we based the first model on sgd [17, and the second one on an SVM implementation called LibSVM [3]. The neural network we used for testing was a multi-layer perceptron (MLP) classifier [17] with one hidden layer with 100 perceptrons in the hidden layer. Last, for Random Forests we tested two different implementations. One which averages the results of every tree [17, and another one based on gradient boosting [4]. 
Table 2. Tested text classifiers

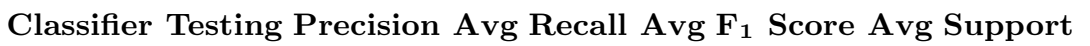

\begin{tabular}{lllll}
\hline $\begin{array}{l}\text { LibLinear } \\
\text { Logistic } \\
\text { Regression }\end{array}$ & 0.89 & 0.86 & 0.84 & 19.3 \\
\hline $\begin{array}{l}\text { SGD Logistic } \\
\text { Regression }\end{array}$ & 0.93 & 0.88 & 0.88 & 20.7 \\
\hline SGD SVM & 0.94 & 0.91 & 0.91 & 20.6 \\
\hline SVM (LibSVM) & 0.95 & 0.92 & 0.92 & 20.9 \\
\hline MLP & 0.86 & 0.76 & 0.76 & 29.9 \\
\hline Random Forest & 0.92 & 0.88 & 0.88 & 19.6 \\
\hline $\begin{array}{l}\text { XGBoost } \\
\text { Random Forest }\end{array}$ & 0.89 & 0.87 & 0.85 & 19.7 \\
\hline
\end{tabular}

As shown in Table 2, most classifiers performed well. Support vector machines shine in cases where the feature vectors have high dimensionality, which is the case for text classification [17]. Logistic Regression models share many advantages with SVMs, and performed well enough. With regard to random forests, even though they both performed well, they need to be configured properly to avoid overfitting. The MLP model performed the worst, likely due to the small dataset we used for training the model. With a larger training dataset, we believe that the results would have been better. Given the current results and the theoretical advantages brought by SVMs, we opted for using the LibSVM model.

\section{Evaluation}

After we have chosen the appropriate feature extraction and text classification algorithms, we defined a set of tests. Our goal is to verify that our approach can identify correctly MUD and BACnet devices. First, we created 2 different training datasets in order to train the classification models. One dataset contains texts for 10 categories of general consumer IoT devices. The other dataset contains these 10 categories of texts, plus 2 categories specific for industrial IoT devices. The final categories are:

- General devices Alarm, Camera, Doorbell, Electric Control, Health Monitor, House Environment Monitor, Light, Media Player, Motion Sensor, Speaker.

- Industrial devices Industry Environment Sensor, Industry Controller.

Additionally, we have created 2 testing datasets. The first dataset contains 24 general consumer IoT devices, in the form of MUD files generated by Hamza et al. [12. The second dataset contains the first dataset items, plus 9 industry IoT devices in the form of BACnet objects. This led to the following tests: 
- MUD -: Testing MUD devices. Training dataset contains only texts and categories of general IoT devices.

- MUD +: Testing MUD devices. Training dataset contains texts and categories of both general and industry IoT devices.

- BACnet +: Testing BACnet devices. Training dataset contains texts and categories of both general and industry IoT devices.

The MUD - test evaluates how well the implementation performs when we only consider MUD devices and categories of devices targeted at general consumers. As an extension, with MUD + we evaluated the same set of IoT devices, but we expanded the categories to include industrial IoT devices.

We did not perform BACnet - tests: with only two categories for industrial IoT devices, the training dataset would have been too small. Last, in BACnet + we tried to identify BACnet-enabled devices using the same categories we used for MUD +. In this way, we were also able to test the generalisability of our approach.

In the first implementation phase, we extract relevant keywords from MUD and BACnet devices, we automatically feed them to search engines (i.e., Google and Bing), and we collect the resulting texts. Then, thanks to the classification model, we classify each text with a score between 0.0 and 1.0 , and we choose the highest scoring one for classifying the device. We have also defined a minimum threshold for discarding every irrelevant text. In case that all the texts score below this threshold, we consider the device non-classified. Initially this threshold was set to 0.1 .

Initial tests gave an accuracy of $75 \%$ for MUD +, $83 \%$ for MUD -, and $33 \%$ for BACnet + . The small dataset we used, as well as the similarities between some categories, negatively affected the result. In particular, some device types were similar in terms of features and some categories shared the same vocabulary. Due to these similarities, the classification scoring would often fail. To alleviate these issues, first we increased the dataset size. Also, we further manually cleaned the texts, in order to remove irrelevant terms.

Some problems also derived from basing the classification on the highest scoring text. Therefore, we opted for classifying each text and then accumulating the score towards each category. To increase separation between higher and lower scoring texts, each classification score is squared. We named this approach Cumulative Squared Scoring (CSS). The CSS approach achieved higher accuracy for both MUD and BACnet, as shown in Table 3. For both MUD tests one device classification was discarded (marked as "No Classification"). This was due to similarities between categories, leading to a classification falling below our pre-defined threshold.

\section{$6 \quad$ Legal Issues}

In our first PoC, we scraped the URLs retrieved from the search engines. This was a problem, since scraping often breaches the websites' terms of service (TOS). Many lawsuits regarding scraping have been filed, with varying outcomes [2]. 
Table 3. Results obtained with CSS and a cleaned data set

\begin{tabular}{lccc} 
CSS & \multicolumn{3}{c}{ MUD - MUD + BACnet +} \\
\hline Accuracy & 0.88 & 0.88 & 0.89 \\
\hline No Classification & 0.04 & 0.04 & 0.00 \\
\hline Average Classification Score & 1.17 & 1.09 & 1.22 \\
\hline
\end{tabular}

There are several cases in the United States, where businesses scraped competitors' websites for financial gain. Such lawsuits ended in favour of the scraped party. Other cases focused on whether or not freely public data should be scrapable ${ }^{4}$. Overall, there seems to be huge variability about the outcomes, depending on specific laws for each country.

In order to minimise any potential legal issue, we modified our implementation. Both Bing and Google return small snippets of text that summarise the contents of the indexed web pages. We decided to use the snippets directly, sidestepping the scraping phase. Our initial dataset were also created through scraping. We replaced all the problematic texts with other texts, scraped from more permissive websites. After testing various thresholds for discarding texts, a new threshold of 0.2 was used, rather than 0.1 . For the sake of clarity, we called this approach TOS. Snippets not only protected us from TOS issues, but also improved our results. As we can see in Table 4, the accuracy improved, compared to Table 3, and the average classification score is higher for each and every item.

Table 4. Results obtained with search engines snippets, cumulative scoring, and a cleaned data set, denoted TOS

\begin{tabular}{lccc} 
TOS & \multicolumn{3}{c}{ MUD } \\
\hline Accuracy & 0.96 & 0.88 & 0.89 \\
\hline No Classification & 0.00 & 0.04 & 0.00 \\
\hline Average Classification Score & 2.53 & 1.82 & 1.63 \\
\hline
\end{tabular}

Moreover, by using snippets and avoiding the scraping phase, the average run time was dramatically reduced. As shown in Table 5 , the average run time for identifying MUD devices is $10 \times$ shorter with snippets. Similarly, the average run time for BACnet devices is $7 \times$ shorter. In terms of devices correctly identified, our final TOS solution allowed us to correctly identify almost $90 \%$ of our MUD and BACnet devices, as shown in Table 6. In Table 77, we provide the detailed list of which devices we were able to correctly identify, and which ones not. In the third column we specified the actual type of device, while in the fourth column we report the output of our classification proof-of-concept. While three of the

4 https://www.tripwire.com/state-of-security/featured/hiq-v-linkedincontrols-publicly-available-data/ 
four failed attempts were plain wrong classifications, one of the devices (i.e., the Blipcare BP Meter) were not classified at all. As aforementioned, some categories are considerably similar to each other; in this particular case, the device scored equally in different categories and failed to meet the minimum threshold we set.

With regard to increased accuracy and classification score shown in Table 4 , we give credit to the relevancy and conciseness of the snippets returned from the search engines. Both Bing and Google have methods for summarising the websites through machine learning algorithms, trained to extract the most relevant words from long texts. The results we obtained indicate that the snippets accurately portray the websites' contents, providing relevant text and very little noise. This also shows that more text does not entail better results. However, if a short text (such as a snippet) contains noise, the classification is affected to a higher degree than if it were dealing with a longer text.

Table 5. Average time required for classifying devices with CSS and TOS

\begin{tabular}{lcc} 
Device & CSS (s) & TOS (s) \\
\hline MUD & 20.24 & 2.78 \\
\hline BACnet & 48.30 & 7.62 \\
\hline
\end{tabular}

Table 6. Results obtained with TOS technique

\begin{tabular}{lc} 
Protocol & Devices Identified (\%) \\
\hline MUD & 87.5 \\
\hline BACnet & 88.9 \\
\hline Total & 87.9 \\
\hline
\end{tabular}

\section{Discussion}

While our implementation performs well, there is room for improvement. Typically, training data sets contain several thousands of texts. However, in our experiment we had to manually create our training dataset, resulting in 5000 to 10000 words per category. This shortage impacted the choice of the classification model, as a larger dataset might have lead to different test results. Moreover, the testing dataset does not have a consistent amount of devices across classes. The test set has 24 MUD devices but only 9 BACnet devices, making some categories not well represented.

There is also variability in performance, depending on the MUD files and the BACnet objects. As we discussed before, we extract the first set of keywords from specific fields. In the case of MUD, the systeminfo field is optional (refer to the specification 5 so it might not be possible to identify the device with our approach. The same goes for BACnet: the descriptive fields in the device object do not have specific requirements, which could lead to potential issues.

Furthermore, our solution is tied to the correctness of the search engines results. If search engines provide text that is irrelevant or misleading, the classification of the device type might be affected. Let us consider the case of Google

\footnotetext{
5 https://datatracker.ietf.org/doc/rfc8520/
} 
Table 7. Final results obtained with our identification technique

\begin{tabular}{|c|c|c|c|c|}
\hline Protocol & Device Name & Type & Predicted Type & Result \\
\hline MUD & Amazon Echo & Speaker & Speaker & Pass $\checkmark$ \\
\hline MUD & August Doorbell & Doorbell & Doorbell & Pass $\checkmark$ \\
\hline MUD & Awair Air Quality & $\begin{array}{l}\text { House Environment } \\
\text { Monitor }\end{array}$ & $\begin{array}{l}\text { House Environment } \\
\text { Monitor }\end{array}$ & Pass $\checkmark$ \\
\hline MUD & Belkin Camera & Camera & Camera & Pass $\checkmark$ \\
\hline MUD & Blipcare BP Meter & Health Monitor & No Classification & Fail $x$ \\
\hline MUD & Canary Camera & Camera & Camera & Pass $\checkmark$ \\
\hline MUD & Chromecast Ultra & Speaker & Speaker & Pass $\checkmark$ \\
\hline MUD & Dropcam & Camera & $\begin{array}{l}\text { House Environment } \\
\text { Monitor }\end{array}$ & Fail $x$ \\
\hline MUD & Phillips Hue Bulb & Light & Light & Pass $\checkmark$ \\
\hline MUD & iHome Power Plug & Electric Control & Electric Control & Pass $\checkmark$ \\
\hline MUD & Lifx Bulb & Light & Light & Pass $\checkmark$ \\
\hline MUD & Nest Smoke Sensor & $\begin{array}{l}\text { House Environment } \\
\text { Monitor }\end{array}$ & $\begin{array}{l}\text { House Environment } \\
\text { Monitor }\end{array}$ & Pass $\checkmark$ \\
\hline MUD & Netatmo Camera & Camera & Camera & Pass $\checkmark$ \\
\hline MUD & Netatmo Weather & $\begin{array}{l}\text { House Environment } \\
\text { Monitor }\end{array}$ & $\begin{array}{l}\text { House Environment } \\
\text { Monitor }\end{array}$ & Pass $\checkmark$ \\
\hline MUD & Ring Doorbell & Doorbell & Doorbell & Pass $\checkmark$ \\
\hline MUD & Samsung Smart Cam & Camera & Camera & Pass $\checkmark$ \\
\hline MUD & TP Link Camera & Camera & Camera & Pass $\checkmark$ \\
\hline MUD & TP Link Plug & Electric Control & Electric Control & Pass $\checkmark$ \\
\hline MUD & Triby Speaker & Speaker & Speaker & Pass $\checkmark$ \\
\hline MUD & Wemo Motion & Motion Sensor & Motion Sensor & Pass $\checkmark$ \\
\hline MUD & Wemo Switch & Electric Control & Electric Control & Pass $\checkmark$ \\
\hline MUD & Withings Baby Monitor & $\begin{array}{l}\text { House Environment } \\
\text { Monitor }\end{array}$ & Health Monitor & Fail $x$ \\
\hline MUD & Withings Cardio & Health Monitor & Health Monitor & Pass $\checkmark$ \\
\hline MUD & Withings Sleep Sensor & Health Monitor & Health Monitor & Pass $\checkmark$ \\
\hline BACnet & $\begin{array}{l}\text { AROB Universal Room } \\
\text { Controller }\end{array}$ & Industry Controller & Industry Controller & Pass $\sqrt{ }$ \\
\hline BACnet & $\begin{array}{l}\text { BACnet Dewpoint } \\
\text { Transmitter }\end{array}$ & $\begin{array}{l}\text { Industry Environment } \\
\text { Sensor }\end{array}$ & $\begin{array}{l}\text { Industry Environment } \\
\text { Sensor }\end{array}$ & Pass $\checkmark$ \\
\hline BACnet & $\begin{array}{l}\text { BAC-RI Room Interface } \\
\text { Module }\end{array}$ & Industry Controller & Industry Controller & Pass $\checkmark$ \\
\hline BACnet & $\begin{array}{l}\text { BACnet Room Pressure } \\
\text { Monitor }\end{array}$ & $\begin{array}{l}\text { Industry Environment } \\
\text { Sensor }\end{array}$ & Health Monitor & Fail $x$ \\
\hline BACnet & $\begin{array}{l}\text { SRI-70 Analogue Room } \\
\text { Interfaces }\end{array}$ & Industry Controller & Industry Controller & Pass $\checkmark$ \\
\hline BACnet & CDD3 CO2 Detector & $\begin{array}{l}\text { Industry Environment } \\
\text { Sensor }\end{array}$ & $\begin{array}{l}\text { Industry Environment } \\
\text { Sensor }\end{array}$ & Pass $\checkmark$ \\
\hline BACnet & $\begin{array}{l}\text { CDR-BAC Room } \\
\text { CO2/Temp Sensor }\end{array}$ & $\begin{array}{l}\text { Industry Environment } \\
\text { Sensor }\end{array}$ & $\begin{array}{l}\text { Industry Environment } \\
\text { Sensor }\end{array}$ & Pass $\checkmark$ \\
\hline BACnet & $\begin{array}{l}\text { SRC-100 Series Zone } \\
\text { Controllers }\end{array}$ & Industry Controller & Industry Controller & Pass $\checkmark$ \\
\hline BACnet & $\begin{array}{l}\text { Touchplate Light } \\
\text { Controller }\end{array}$ & Industry Controller & Industry Controller & Pass $\checkmark$ \\
\hline
\end{tabular}

bombing, the act of boosting a website pagerank for unrelated search terms, by massively linking from external webpages [1]. As a main side effect of this practice, search results are polluted with a considerable amount of noise. If we try to classify a security camera, and an adversary manipulated the results such that 
websites describing lamps (instead of cameras) are showed, our algorithm might lead to a misclassification. In turn, the misclassification might lead to security issues.

\section{Related Work}

In this paper, our goal is to infer the type of IoT devices. Manufacturer Usage Descriptions (MUD) [13] is an IETF specification (RFC8520), in which manufacturers specify which hosts and ports their devices need for operating correctly. Even though MUD does not allow exhaustive behavioural descriptions, MUD files can be used for profiling IoT devices without time-consuming phases for collecting devices' traffic packets.

Hamza et al. 12] proved that is possible to convert pcap traces to behavioural description files, such as MUD. The authors describe their conversion tool and showcase its use by creating MUD profiles for 28 popular IoT devices. These devices are a good base for testing our implementation. Other works show that is possible to detect a device type from its network traffic [20. This is done by monitoring different variables, such as the amount of data, communication patterns, idle time, port numbers, DNS queries, and so on. By collecting months of data, the authors were able to identify various devices through their network traffic.

From a larger perspective, researchers proposed different approaches for achieving reliable fingerprints of IoT devices. Yang et al. 21 proposed a triplet label (type,vendor,product) for identifying IoT devices, and a fingerprint generation approach based on neural networks. Meidan et al. [16] proposed ProfilloT, a tool based on supervised learning, capable of identifying accurately IoT devices. However, it is unclear if such approaches would be feasible for achieving near real-time fingerprinting, which is a critical characteristic for managing IoT devices joining a network for the first time.

\section{Conclusion}

In this paper, we have proposed a technique for device identification which combines natural language processing, text classification, and web search engines. First, we tested different feature extraction and text classification models, opting for combining TF-IDF and Support Vector Machines. Second, we identified two web search engines and, in order to avoid terms of services issues, we opted for gathering data from the result snippets instead of scraping websites.

Then, we implemented a PoC and we applied it to various IoT devices. With a success rate of $87.5 \%$ for MUD devices, and $88.9 \%$ for BACnet devices, our experiments showed that not only it is possible to identify devices' types, but it can also be done in a matter of a few seconds. In future works, we plan to extend our PoC to Shodan (a web search engine dedicated to IoT devices), and to assess the robustness of our approach when evaluating non-IoT devices. 


\section{References}

1. Adali, S., Liu, T., Magdon-Ismail, M.: An Analysis of Optimal Link Bombs. Theoretical Computer Science 437, 1-20 (2012)

2. Broucke, S.v., Baesens, B.: Practical Web Scraping for Data Science: Best Practices and Examples with Python. Apress, New York, NY (2018)

3. Chang, C.C., Lin, C.J.: LIBSVM: A Library for Support Vector Machines. ACM Trans. Intell. Syst. Technol. 2(3) (May 2011)

4. Chen, T., Guestrin, C.: XGBoost: A Scalable Tree Boosting System. In: Proceedings of the 22Nd ACM SIGKDD International Conference on Knowledge Discovery and Data Mining. KDD '16, ACM, New York, NY, USA (2016)

5. De Donno, M., Dragoni, N., Giaretta, A., Spognardi, A.: DDoS-Capable IoT Malwares: Comparative Analysis and Mirai Investigation. Security and Communication Networks 2018, 1-30 (02 2018)

6. Dragoni, N., Massacci, F., Walter, T., Schaefer, C.: What the Heck is this Application Doing? A Security-by-Contract Architecture for Pervasive Services. Computers \& Security 28(7), 566-577 (2009)

7. Dragoni, N., Giaretta, A., Mazzara, M.: The Internet of Hackable Things. In: Proceedings of 5th International Conference in Software Engineering for Defence Applications. pp. 129-140. Springer International Publishing, Cham (2018)

8. Fan, R.E., Chang, K.W., Hsieh, C.J., Wang, X.R., Lin, C.J.: LIBLINEAR: A Library for Large Linear Classification. J. Mach. Learn. Res. 9 (Jun 2008)

9. Giaretta, A., Dragoni, N., Massacci, F.: IoT Security Configurability with Securityby-Contract. Sensors 19(19), 4121 (2019)

10. Giaretta, A., Dragoni, N., Massacci, F.: Protecting the Internet of Things with Security-by-Contract and Fog Computing. In: 2019 IEEE 5th World Forum on Internet of Things (WF-IoT). pp. 1-6. IEEE, New York, NY (2019)

11. Haakenstad, L.K.: The Open Protocol Standard for Computerized Building Systems: BACnet. In: Proceedings of the 1999 IEEE International Conference on Control Applications. vol. 2, pp. 1585-1590. IEEE, New York, NY (Aug 1999)

12. Hamza, A., Ranathunga, D., Gharakheili, H.H., Roughan, M., Sivaraman, V.: Clear As MUD: Generating, Validating and Applying IoT Behavioral Profiles. In: Proceedings of the 2018 Workshop on IoT Security and Privacy. IoT S\&P '18, ACM, New York, NY, USA (2018)

13. Lear, E., Weis, B.: Slinging MUD: Manufacturer Usage Descriptions: How the Network Can Protect Things. In: International Conference on Selected Topics in Mobile Wireless Networking (MoWNeT). pp. 1-6. IEEE, New York, NY (2016)

14. Lim, S., Kwon, O., Lee, D.H.: Technology Convergence in the Internet of Things (IoT) Startup Ecosystem: A Network Analysis. Telematics and Informatics 35(7), 1887-1899 (2018)

15. Mahmood, Z.: Fog Computing: Concepts, Frameworks and Technologies. Springer International Publishing, Cham (2018)

16. Meidan, Y., Bohadana, M., Shabtai, A., Guarnizo, J.D., Ochoa, M., Tippenhauer, N.O., Elovici, Y.: Profiliot: A machine learning approach for iot device identification based on network traffic analysis. In: Proceedings of the Symposium on Applied Computing. pp. 506-509. SAC '17, Association for Computing Machinery, New York, NY, USA (2017)

17. Pedregosa, F., Varoquaux, G., Gramfort, A., Michel, V., Thirion, B., Grisel, O., Blondel, M., Prettenhofer, P., Weiss, R., Dubourg, V., Vanderplas, J., Passos, A., Cournapeau, D., Brucher, M., Perrot, M., Duchesnay, E.: Scikit-learn: Machine 
Learning in Python. Journal of Machine Learning Research 12(85), 2825-2830 (2011)

18. Rayes, A., Salam, S.: Internet of Things From Hype to Reality: The Road to Digitization. Springer International Publishing, Cham (2019)

19. Román-Castro, R., López, J., Gritzalis, S.: Evolution and Trends in IoT Security. Computer 51(7), 16-25 (July 2018)

20. Sivanathan, A., Gharakheili, H.H., Loi, F., Radford, A., Wijenayake, C., Vishwanath, A., Sivaraman, V.: Classifying iot devices in smart environments using network traffic characteristics. IEEE Transactions on Mobile Computing 18(8), 1745-1759 (2019)

21. Yang, K., Li, Q., Sun, L.: Towards automatic fingerprinting of iot devices in the cyberspace. Computer Networks 148, 318-327 (2019) 